\title{
Lateralization of spatial categories: A comparison of verbal and visuospatial categorical relations
}

\author{
Ineke J. M. van der Ham and Albert Postma \\ Utrecht University, Utrecht, The Netherlands
}

\begin{abstract}
Many reports show that spatial relations between and within objects show differences in hemispheric lateralization. Coordinate, metric relations concerning distances are processed with a right-hemisphere advantage, whereas a left-hemisphere advantage is thought to be related to categorical, abstract relations (Kosslyn, 1987). Kemmerer and Tranel (2000) argued that the left-hemisphere advantage for categorical processing might apply only for verbal spatial categories, however, whereas a right-hemisphere advantage is related to visuospatial categories. To test this idea, we examined categorical processing for stimuli in both verbal and visuospatial formats, with a visual half-field, match-to-sample design. In Experiment 1, we manipulated the format of the second stimulus to compare response patterns for both verbal and visuospatial stimuli. In Experiment 2, we varied the expectancy of the format of the second stimulus, allowing for an assessment of strategy use. The results showed that a left-hemisphere advantage was related to verbal stimulus format only, but not in all conditions. A right-hemisphere advantage was found only with a visuospatial expectancy, visuospatial format, and brief interval. The theory we present to explain these results proposes that the lateralization related to basic categorical processing can be strongly influenced by verbal characteristics and, to some extent, by additional coordinate processing. The lateralization measured in such cases does not represent lateralization related purely to categorical processing, but to these additional effects as well. This stresses the importance of careful task and stimulus design when examining categorical processing in order to reduce the influence of those additional processes.
\end{abstract}

Spatial relation processing is a vital element in the perception of our surroundings. It provides us with information about where things are and how we can interact with them spatially. These relations have often been described as belonging to two general classes: categorical, relative, abstract labels like "left of," and coordinate, metric descriptions like "one meter apart" (Kosslyn, 1987). Along with these definitions, Kosslyn and colleagues (Kosslyn, 1987; Kosslyn et al., 1989) proposed a difference between the two types of spatial relations with regard to hemispheric lateralization; categorical relations show a left-hemisphere advantage, whereas coordinate relation processing depends more on a right-hemisphere circuitry (see, e.g., Hellige \& Michimata, 1989; Laeng \& Peters, 1995; Rybash \& Hoyer, 1992; see also Jager \& Postma, 2003). Notably, the right-hemisphere advantage for coordinate processing has been supported by numerous reports, whereas the lefthemisphere advantage for categorical processing has been smaller or absent in some studies (see Laeng, Chabris, \& Kosslyn, 2003). So far, factors such as individual differences like gender (Rybash \& Hoyer, 1992) and variation in difficulty (see, e.g., Martin, Houssemand, Schiltz, Burnod, \& Alexandre, 2008) have been regarded as potential causes for these inconsistencies. The effects of both of these factors are still a matter of debate, however, and they do not seem clearly linked to the inconsistencies of categorical processing in particular.
Kemmerer and Tranel (2000) provided a theory of how task demands might determine the direction of lateralization. They suggested that spatial categories can be divided into two different classes. Their results indicated that only the use of linguistic categories is impaired by lefthemisphere damage, whereas a right-hemisphere lesion disturbed the processing of solely perceptual, or visuospatial categories. Using an elaborate battery of tests, they illustrated this double dissociation by contrasting one left-hemisphere patient with one right-hemisphere patient. Their linguistic tests focused on the use of spatial prepositions, whereas the perceptual tests - conventional neuropsychological tests - are considered to not have any verbal characteristics. These results should be considered with some caution, however, because in perceptual category tasks there appears to be some additional demand on coordinate relation processing, which, as reported above, is commonly related to a right-hemisphere advantage. Also, the idea of perceptual categories without any linguistic characteristics seems improbable, since they are defined as having the potential to be described in abstract propositional terms. The partially conflicting theories of Kosslyn (1987) and Kemmerer and Tranel thus raise the question of whether these two types of categorical representations are truly different in how they are lateralized in the brain or are merely expressions of the same construct in different representational formats. The critical distinction between 
these two lines of thought is whether perceptual or visuospatial category processing can be dissociated from verbal category processing by right-hemisphere involvement (Kemmerer \& Tranel, 2000) or not (Kosslyn, 1987).

The goal of the present study was to explore this issue further and to focus on the type of task that measures lateralization of categorical relations. We aimed to gain more insight into why the theorized left-hemisphere advantage is not always clearly present. In two experiments, we concentrated on the nature or the format of the stimuli that were used to manipulate the verbal and visuospatial nature of a categorical task. In this way, we investigated whether the difference between verbal and visuospatial processing caused by stimulus format can account for a difference in direction of lateralization. As stated before, we do not believe that visuospatial categories exist without any possibility of linguistic coding, but by manipulating the stimulus formats, we were able to compare highly verbal and highly visuospatial conditions properly.

In short, along with the question of how the format of stimuli affects lateralization for categorical trials, we focused on the possibility of separating verbal and visuospatial categories with regard to hemispheric processing. To address both issues, we adapted the categorical version of the cross-dot task (van der Ham, Raemaekers, van Wezel, Oleksiak, \& Postma, 2009; van der Ham, van Wezel, Oleksiak, \& Postma, 2007). In Experiment 1, we explored the lateralization effects in a basic setting similar to that in the original cross-dot design, and we looked further into the effects of strategy use in Experiment 2 by changing the expectancy of the stimulus format.

\section{EXPERIMENT 1}

For Experiment 1, we adapted the cross-dot task reported in previous studies (van der Ham et al., 2009; van der Ham et al., 2007). In the original categorical version of the cross-dot task, subjects compared two sequentially presented stimuli consisting of a plus-shaped cross with a dot located somewhere around the cross. They were asked to determine whether or not the dots in both stimuli matched with regard to the quadrant they were in. Each quadrant represented a spatial category indicated by the horizontal and vertical axes, so the task entailed determining whether the first and second dot belonged to the same spatial category or not. Coordinate position information could be ignored; only categorical information was required for a correct answer. This task entailed the lateral presentation of the second stimulus in each trial. Since performance for stimuli that were presented to one visual half-field could be related to the contralateral hemisphere (see, e.g., Bourne, 2006), the design of this behavioral experiment allowed for assessment of hemispheric processing.

In the present experiment, we varied the format of the second stimulus. It consisted of a visuospatial depiction that was identical in composition to the stimuli used in the original experiment, a more abstract visuospatial reflection of the cross-dot stimuli, or a verbal description. The abstract visuospatial stimuli also consisted of the cross, but one whole quadrant was highlighted in gray, instead of one of the quadrants containing a single dot. This abstract version was added because it can be considered a combination of the visuospatial and verbal formats; it is a truly visuospatial depiction but without any irrelevant, coordinate position information within a category, which was also absent for the verbal stimuli. These verbal stimuli consisted of a verbal label describing the quadrant the dot was in (a combination of the words left/right and top/bottom). Each format was featured in $33 \%$ of the trials, in random order. In this way, we could assess the lateralization patterns that were related to each format and see whether visuospatial and verbal categories were processed differently.

An additional feature of the task was that the retention interval between the first and second stimuli was varied. In line with previous studies (e.g., van der Ham et al., 2007), these intervals were set to $500 \mathrm{msec}, 2,000 \mathrm{msec}$, and 5,000 msec. Self-reports of subjects in these studies indicated that their preferred approach to the comparisons varied for the different interval lengths. Generally, they preferred a more visuospatial approach when the interval was $500 \mathrm{msec}$, but most of them reported that they verbalized the stimuli for the 2,000-msec and 5,000-msec intervals. We have therefore maintained this variable in order to assess whether strategic recoding differences also occurred in the present experiment.

If the distinction made by Kemmerer and Tranel (2000) is correct, then we would expect a left-hemisphere advantage for the verbal trials and a right-hemisphere advantage for the visuospatial and the abstract visuospatial trials. If the previous self-reports are a correct reflection of subjects' behavior, we might find an increase in left-hemisphere performance with longer intervals because of an increase in verbalization of the stimuli. If the more traditional view of categorical processing initially suggested by Kosslyn (1987) is valid, we would expect a left-hemisphere advantage for all three formats, since they all address the categorical relations in the stimuli. With regard to retention interval, an overall left-hemisphere advantage would theoretically be expected; in our previous data, however, we found this advantage at a significant level for only the 500 -msec condition (van der Ham et al., 2009; van der Ham et al., 2007). This can possibly be explained by decay of spatial information over time; it might not be significant for the longer intervals, but according to the Kosslyn view we would not expect a change in direction of lateralization over time.

\section{Method}

Subjects. Thirty-nine subjects were included in the experiment (20 female, 19 male), with a mean age of $22.4(S D=3.01)$. One male subject was excluded from further analysis because of performance at chance level. Right-handedness was ensured for all subjects by administering the Edinburgh Handedness Inventory (Oldfield, 1971), and only native Dutch-speaking subjects were included. All subjects were neurologically healthy and had normal or corrected-to-normal vision.

Design and Procedure. This experiment was an adapted version of the cross-dot experiment (see van der Ham et al., 2009; van der Ham et al., 2007) in which subjects were asked to compare two sequentially presented stimuli. The cross-dot stimulus design used in this experiment is shown in Figure 1, in which a cross with all possible dot positions is depicted. Only the categorical instruction was used; the dot position with regard to the cross indicated one 


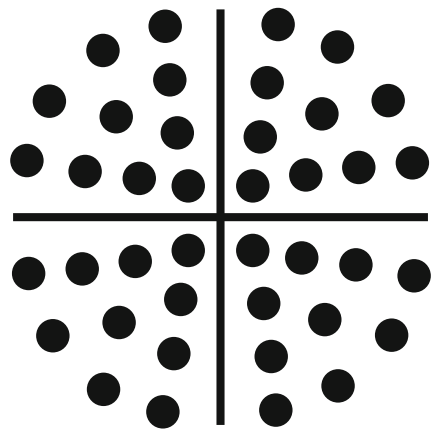

Figure 1. All possible dot positions of the cross-dot stimuli. Note that only one dot position was visible in a single stimulus.

of the four possible categories (top left, top right, bottom left, bottom right), which subjects were asked to compare between the two stimuli. If the dot was in the same quadrant for both the first and second stimuli, then a "match" response should be given. If they were in different quadrants, then a "no match" response was the correct answer. The adaptation in this version of the task comprised a change in the format of the second stimulus. There were three possibilities: visuospatial, abstract visuospatial, and verbal. Examples of these options are depicted in Figure 2. The dimensions of visuospatial and abstract visuospatial images were $90 \times 90$ pixels, and those of the verbal images were $105 \times 22$ pixels. The visuospatial stimuli were identical in composition to the first stimuli: a cross with a dot. For these second stimuli, the dot was never at exactly the same position as in the first image. The abstract visuospatial version consisted of a gray square filling an entire quadrant of the square; if the gray area of the square of the second stimulus included the location of the first stimulus's dot position, this would be a match. These stimuli were similar to the visuospatial stimuli, but they contained less (irrelevant) positional information and were designed as an "abstract" depiction of the verbal stimuli, containing the same amount of information as the verbal descriptions. The descriptions "top left," "top right," "bottom left," and "bottom right" (in Dutch: "links boven," "rechts boven," "links onder," and "rechts onder") were used for the verbal format. Subjects were asked to compare the relative dot position to the text shown and give a "match" or "no match" response in the same manner as for the visuospatial stimuli.

A single trial sequence proceeded as follows: a colored square cuing the start of a trial ( $500 \mathrm{msec}$ in duration), a fixation cross $(500 \mathrm{msec})$, the first stimulus $(150 \mathrm{msec}$, presented centrally), a blank screen $(0,1,500$, or $4,500 \mathrm{msec})$, a fixation cross $(500 \mathrm{msec})$, the second stimulus $\left(150 \mathrm{msec}\right.$, presented laterally at $2.5^{\circ}$ from the center of the stimulus to the center of the screen), and a blank screen during which a response could be given $(2,000 \mathrm{msec})$. Only the second stimulus was presented laterally, with the brief duration of $150 \mathrm{msec}$ to prevent eye movements, as is commonly used for visual half-field studies (Bourne, 2006). In total, 360 trials were presented in nine blocks of 40 trials, divided equally over all conditions. Trials were blocked according to interval length (500 $\mathrm{msec}, 2,000 \mathrm{msec}$, and 5,000 msec), whereas format (visuospatial, abstract visuospatial, and verbal) and visual field (left and right) were presented in random order.
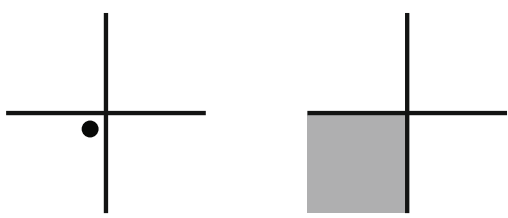

Links Onder

Figure 2. Examples of all three possible formats of the second stimulus in a trial: visuospatial, abstract visuospatial, and verbal.
Subjects were seated in front of a computer screen $(1,048 \times$ 768 pixels), with a chinrest $50 \mathrm{~cm}$ away from the screen to keep their head fixated at the center of the screen. The experiment was presented with Presentation software (Neurobehavioral Systems). Responses were given by means of a four-button response box designed to reduce any possible lateralization effects of motor activity; both index fingers were used for a "match" response, whereas both middle fingers were used for a "no match" response. Prior to the experiment, subjects signed an informed consent form and were instructed orally. Examples of the stimuli were shown by the experimenter, and the experiment started with a brief summary of the instructions and some practice trials.

Data analysis. All error rates (ERs) and response times (RTs) were registered. RTs of incorrect trials and those over 2,000 msec were excluded from the analyses. A repeated measures general linear model (GLM) was used to analyze main and interaction effects. The factors involved were format (3), retention interval (3), and visual field (2). Significant interaction effects were followed up with Bonferroni-corrected post hoc tests.

\section{Results}

The average ERs and RTs are reported in Table 1. The analysis of ER showed a significant main effect for format $[F(2,36)=39.47, p<.000]$ and for visual field $[F(1,37)=$ $10.81, p<.01]$. The average number of errors was significantly higher for the verbal format than for the abstract visuospatial and visuospatial formats. Overall, a lower ER was found for stimuli that were presented to the right visual field/left hemisphere (RVF/LH); however, a significant format $\times$ visual field interaction $[F(2,36)=7.42, p<.01]$ indicated that the main effect of visual field was affected by format. Follow-up tests for each format showed that a significant effect of visual field was restricted to the verbal format $[F(1,37)=10.51, p<.01]$; fewer errors were made during RVF/LH stimulus presentation.

The RTs showed significant main effects for format $[F(2,36)=198.18, p<.001]$, retention interval $[F(2,36)=$ $3.67, p<.05]$, and visual field $[F(1,37)=11.11, p<$ .01]. The fastest RTs were those to abstract visuospatial stimuli, followed by those to visuospatial stimuli, and the slowest RTs were those to verbal stimuli. Faster RTs were also found for the 2,000-msec interval than for the 5,000msec interval, and RTs were faster for the RVF/LH than for the left visual field/right hemisphere (LVF/RH). Significant interaction effects were found for format $X$ retention interval $[F(4,34)=4.64, p<.005]$ and for format $\times$ visual field $[F(2,36)=15.63, p<.001]$. Follow-up tests showed a significant main effect for retention interval for both the visuospatial format $[F(2,36)=6.12, p<.01]$ and the abstract visuospatial format $[F(2,36)=6.96, p<.01]$. In both cases, the 5,000-msec retention interval showed significantly higher RTs. A significant main effect of visual field was found for the verbal format only $[F(1,37)=$ $21.06, p<.001]$, indicating lower RTs for stimuli presented to the RVF/LH.

\section{Discussion}

This first experiment was designed to examine the possibility of distinguishing verbal and visuospatial categorical processing on the basis of lateralization patterns. Kemmerer and Tranel (2000) favor this distinction and argue that only verbal categories show a left-hemisphere advantage, 
Table 1

Mean Error Rates (\%) and Response Times (in Milliseconds) for the 500-msec, 2,000-msec, and 5,000-msec Retention Intervals for Experiment 1

\begin{tabular}{|c|c|c|c|c|c|c|c|c|c|c|c|c|c|}
\hline \multirow[b]{3}{*}{ Format } & \multirow{3}{*}{$\begin{array}{l}\text { Visual } \\
\text { Field }\end{array}$} & \multicolumn{4}{|c|}{$500 \mathrm{msec}$} & \multicolumn{4}{|c|}{$2,000 \mathrm{msec}$} & \multicolumn{4}{|c|}{$5,000 \mathrm{msec}$} \\
\hline & & \multicolumn{2}{|c|}{ Error Rate } & \multicolumn{2}{|c|}{ Response Time } & \multicolumn{2}{|c|}{ Error Rate } & \multicolumn{2}{|c|}{ Response Time } & \multicolumn{2}{|c|}{ Error Rate } & \multicolumn{2}{|c|}{ Response Time } \\
\hline & & $M$ & $\overline{S E M}$ & $M$ & $\overline{S E M}$ & $M$ & $\overline{S E M}$ & $M$ & $\overline{S E M}$ & $M$ & $\overline{S E M}$ & $M$ & $S E M$ \\
\hline \multirow[t]{2}{*}{ Visuospatial } & Left & 2.1 & 0.55 & 722.3 & 18.7 & 2.1 & 0.45 & 729.2 & 22.3 & 0.8 & 0.43 & 763.1 & 26.1 \\
\hline & Right & 2.0 & 0.53 & 739.3 & 20.8 & 1.6 & 0.54 & 730.0 & 22.1 & 1.0 & 0.44 & 768.5 & 26.0 \\
\hline \multirow[t]{2}{*}{ Abstract visuospatial } & Left & 2.1 & 0.59 & 725.8 & 17.8 & 1.8 & 0.46 & 713.4 & 22.1 & 1.6 & 0.40 & 755.8 & 24.6 \\
\hline & Right & 1.7 & 0.69 & 720.2 & 17.3 & 2.2 & 0.47 & 710.4 & 21.6 & 1.3 & 0.58 & 749.9 & 25.8 \\
\hline \multirow[t]{2}{*}{ Verbal } & Left & 9.3 & 1.27 & 946.7 & 30.7 & 9.7 & 1.63 & 940.9 & 28.3 & 9.2 & 1.66 & 924.3 & 33.7 \\
\hline & Right & 7.1 & 1.51 & 903.9 & 27.7 & 5.7 & 0.90 & 885.1 & 27.3 & 5.6 & 1.14 & 908.4 & 27.8 \\
\hline
\end{tabular}

whereas Kosslyn (1987) and others (see Jager \& Postma, 2003; Laeng et al., 2003) suggest a left-hemisphere advantage regardless of the nature of the task.

The analysis of visual-field effects showed that lateralization was present in the present data, but only for the verbal format. It was clearly not present for the visuospatial and abstract visuospatial formats. This pattern supports both views partially; we found a left-hemisphere advantage, but it was limited to the verbal format, as predicted by Kemmerer and Tranel (2000). We did not find any significant right-hemisphere advantages, however, which is favorable to the Kosslyn (1987) view. Taken together, these results indicate that the verbal format seems to be related to a clear left-hemisphere advantage, for the short and intermediate intervals. There was no such effect for the visuospatial and abstract visuospatial formats, but there was no evident right-hemisphere advantage either. One plausible interpretation is that the direction of lateralization was determined by the type of spatial relation (categorical), as proposed by Kosslyn, but that the extentand in this case the significance-of this lateralization was determined by the format of the stimulus. This supports the notion of a distinction between visuospatial and verbal categories, as proposed by Kemmerer and Tranel, but one that is less rigid; this distinction is most likely not based on opposing hemispheric advantages. It should be noted that the clear left-hemisphere advantage found for the verbal format might have been amplified by a lefthemisphere involvement in reading the second stimulus.

We also demonstrated that the verbal format is clearly more difficult than the visuospatial and abstract visuospatial formats. This effect is likely due to the need to switch from a visuospatial to a verbal format, which does not occur for the other two formats. We also found that the abstract visuospatial format shows lower RTs than do the visuospatial and verbal formats, and that this effect tends to be stronger for longer intervals. This could be due to a slightly better compatibility with the representation of the first stimulus in memory; since coordinate information decays immediately and a bias toward categories increases over time, this representation is likely to be more abstract with longer intervals (Huttenlocher, Hedges, \& Duncan, 1991; Postma, Huntjens, Meuwissen, \& Laeng, 2006; Werner \& Diedrichsen, 2002). The stimulus representation for the longer intervals is thus most likely more similar to the abstract visuospatial format than to the visuospatial format. Along the same line of rea- soning, we can explain the differential effect of retention interval for the formats. For both the visuospatial and abstract visuospatial formats, we find higher RTs with longer intervals, which is congruent with our previous findings (van der Ham et al., 2007). This indicates that categorical representations are also subject to decay, but not as fast as are coordinate representations. In contrast, the verbal format was unaffected by retention interval, which - along with the overall longer RTs - suggests that a mental transformation of the format of one of the stimuli takes place in order to allow an adequate comparison with the other. We cannot be completely certain which of the two stimuli is transformed, but it is likely to be the second. Since there is only a $33 \%$ chance that a verbal stimulus will appear, a visuospatial encoding of the first stimulus would be most efficient.

The expectancy of the second stimulus's format is an interesting asset of the task design, and it was addressed in a second experiment. Additional evidence for the effect of format of categorical relations on left-hemisphere processing can follow not only from manipulation of the format of the stimuli but also from the format of the mental representation of the stimuli. Experiment 2 was designed to manipulate the expectancy level of stimulus format, changing it from an even distribution into a clear majority of one format over another. A clear expectancy of a specific format affects the most efficient representational format for those trials and therefore allowed us to examine the effects of both visuospatial and verbal mental representations on categorical lateralization (cf. Tversky, 1969, 1974; Noordzij, van der Lubbe, \& Postma, 2005).

\section{EXPERIMENT 2}

In Experiment 1, we used three different stimulus formats under the assumption that the format of the stimulus determines the accompanying representational code (i.e., either visuospatial or verbal); however, the coding strategy preferred by the subjects could also be important. As mentioned before, we have found differences in strategic approach for the cross-dot task on the basis of self-reports (van der Ham et al., 2007). The self-reports indicated differences in strategy between categorical and coordinate processing - more verbal versus more visuospatial, respectively. Also, they showed that, in general, subjects tend to use a more visuospatial strategy for the brief, 500-msec interval, whereas a verbal approach is used for the longer in- 
tervals, regardless of the type of processing. These findings raise the question of whether strategy might also have an effect on lateralization patterns. Would a more verbal strategy be related to a relative left-hemisphere advantage and a visuospatial strategy to a right-hemisphere advantage?

The effect of strategy has been addressed previously in studies focusing on "picture-sentence comparisons." Categorical spatial relations have been studied in verbal and visuospatial formats in these experiments, which have been concerned mainly with the way in which spatial sentences and pictures differ in the mental representations they evoke (e.g., Carpenter \& Just, 1975; Chase \& Clark, 1972) but have never focused on hemispheric lateralization. One of the important findings in this line of research has been that by increasing the linguistic difficulty of the sentences used (e.g., "plus below star" vs. "plus not below star"), the verification times were elevated, indicating that the sentences were represented propositionally and not pictorially (Clark $\&$ Chase, 1972). Elsewhere, however, it has been found that this representational bias may show strong individual differences (MacLeod, Hunt, \& Mathews, 1978). This has led to a series of reports that focused on strategies within this type of experiment by grouping subjects according to their preferred strategy (e.g., Noordzij, van der Lubbe, Neggers, \& Postma, 2004; Reichle, Carpenter, \& Just, 2000; Roberts, Wood, \& Gilmore, 1994).

Noordzij et al. (2005) examined strategy use by changing task parameters instead of grouping subjects according to their preferred strategy on the basis of their behavioral data pattern. They added sentence-sentence comparisons to allow for more verbal and more visuospatial stimulus comparisons. In a match-to-sample paradigm, they set the proportion of the expected format to $80 \%$, and the unexpected format was presented in $20 \%$ of the trials. In half of the trials, a sentence was the expected stimulus format, whereas a picture was expected in the other half. Three possible approaches could be conceived: propositional, pictorial, and strategic. In the propositional and pictorial approaches, the expectancy manipulation would not have an effect on performance, and these two types could be dissociated by testing for relative advantages for either sentences or pictures. Performance with the strategic approach would be affected by expectancy, because subjects would then adjust their strategy to the expected format. The results of the Noordzij et al. (2005) study mostly concurred with the strategic approach. More specifically, the authors argued for a dual representational model. This entails that the sentence is always represented propositionally, because it shows no advantage in performance when it is expected, and an additional pictorial representation is formed only if the context calls for it.

Since this strategic pattern has been found in a situation in which the first stimulus was always a sentence, it raises the question of whether this dual representational model will also generalize to the present picture-picture/ picture-word design used for our cross-dot task. Along with our question as to whether and when categorical processing is left or right lateralized, this motivated us to use the same cross-dot design described for Experiment 1, and to add the expectancy manipulation for the visuospatial and verbal formats. Of central interest, we examined whether the left-hemisphere advantage occurs only when one expects a verbal format for the second stimulus, in line with a strategic propositional approach. It might be that the role of the propositional approach increases with longer retention intervals, moreover, as indicated by the previous self-reports.

We framed our expectations according to the two different theories. The Kemmerer and Tranel (2000) view proposes a left-hemisphere advantage for verbal tasks and a right-hemisphere advantage for visuospatial tasks; therefore, a left-hemisphere advantage should occur with both a verbal stimulus format and a verbal expectancy. In turn, a right-hemisphere advantage should be present with both visuospatial stimulus format and visuospatial expectancy. The two other, incongruent combinations of expectancy and format (verbal-visuospatial and visuospatial-verbal) should not lead to clear lateralization.

The traditional Kosslyn view suggests an overall lefthemisphere advantage that is independent of the second stimulus. No matter what stimulus combination is presented or what type of strategy is used, therefore, one should find a categorical left-hemisphere advantage, never a right-hemisphere advantage. One could argue that a strategic approach would be more likely within this perspective than within the Kemmerer and Tranel (2000) theory. Since the strategic approach requires the flexibility to switch between both strategies according to task demands, this might have a lower cost when both verbal and visuospatial information are processed within the same hemisphere.

\section{Method}

Subjects. Twenty-four subjects ( 12 female, 12 male) participated in the experiment, with a mean age of $21.87(S D=4.1)$. One male subject was excluded because of significantly poor performance (over three standard deviations below group mean). Righthandedness was ensured by means of the Edinburgh Handedness Inventory (Oldfield, 1971). All subjects were neurologically healthy and had normal or corrected-to-normal vision.

Design and Procedure. In this experiment, the format expectancy was altered; instead of an equal distribution of the formats, one format was set to $80 \%$ prevalence and the other to $20 \%$. For this experiment, only the visuospatial and verbal formats were included in order to enable a clear dissociation between the possible verbal and visuospatial strategies. The experiment consisted of two sessions, therefore, performed one directly after the other. The order of the $80 \%$ prevalent format in the two sessions was counterbalanced and was randomly assigned to the subjects.

The trial design was identical to the design used in Experiment 1. Again, the first stimulus was presented centrally and the second laterally, and the retention interval between the two stimuli could be $500 \mathrm{msec}, 2,000 \mathrm{msec}$, or 5,000 msec. The first stimulus was always in the visuospatial, cross-dot format. The format of the second stimulus was either visuospatial (a cross with a dot) or verbal (a description of the quadrant, using left/right and top/bottom), as described for Experiment 1 . One alteration was made to the cross-dot stimuli; instead of a large cross, a smaller cross was used. Arguably, this made the task more sensitive to lateralization effects (see van der Ham et al., 2009).

In total, 720 trials were used-360 in the $80 \%$ visuospatial session, and 360 in the $80 \%$ verbal session. Within each session, the trials were blocked according to the three retention intervals (500 msec, 2,000 $\mathrm{msec}, 5,000 \mathrm{msec}$ ), and visual field (left, right) 
and formats (visuospatial, verbal) were presented randomly within each block. The experimental setup was identical to the setup used for Experiment 1. Subjects received an oral instruction to the task; they were told that one format would be presented more often than the other-and which one that would be-but not the exact percentages. The subjects were shown examples of the stimuli on paper, and they performed a few practice trials before each part of the experiment.

Data analysis. RT and ER data were collected for all trials. RTs of incorrect trials and those over 2,000 msec were excluded from the analysis. A repeated measures GLM was performed, including expectancy (2), format (2), retention interval (3), and visual field (2) as factors. Significant interaction effects were followed up with Bonferroni-corrected post hoc tests.

\section{Results}

The data reflecting strategic approach are given in Figure 3 , and the lateralization patterns are depicted in Table 2 . Significant main effects were found for format $[F(1,22)=$ $16.27, p<.01]$ and visual field $[F(1,22)=8.00, p<.05]$ for the ER data, indicating fewer errors for the visuospatial format than for the verbal format and an overall RVF/
LH advantage. Additionally, significant interaction effects of expectancy $\times$ retention interval $[F(2,21)=5.45, p<$ $.01]$ and format $\times$ visual field $[F(1,22)=7.55, p<.05]$ were found. For the 500-msec and 2,000-msec intervals, the visuospatial expectancy had lower ERs than did the verbal expectancy, but that was reversed for the 5,000msec interval. The RVF/LH advantage was restricted to the verbal format. For the visuospatial format, the two visual fields showed equal ERs.

The RT analysis showed significant main effects for retention interval $[F(2,21)=5.10, p<.05]$, format $[F(1,22)=47.46, p<.001]$, and visual field $[F(1,22)=$ $6.35, p<.05]$. RTs were lower for the 2,000 -msec interval than for the 5,000-msec retention interval, and they were lower for the visuospatial format than for the verbal format. Lower RTs were found for RVF/LH. Significant interactions were found for expectancy $\times$ format $[F(1,22)=$ $138.42, p<.001]$, format $\times$ visual field $[F(1,22)=$ $13.61, p<.01]$, expectancy $\times$ retention interval $\times$ format $[F(2,21)=3.78, p<.05]$, and expectancy $\times$ retention

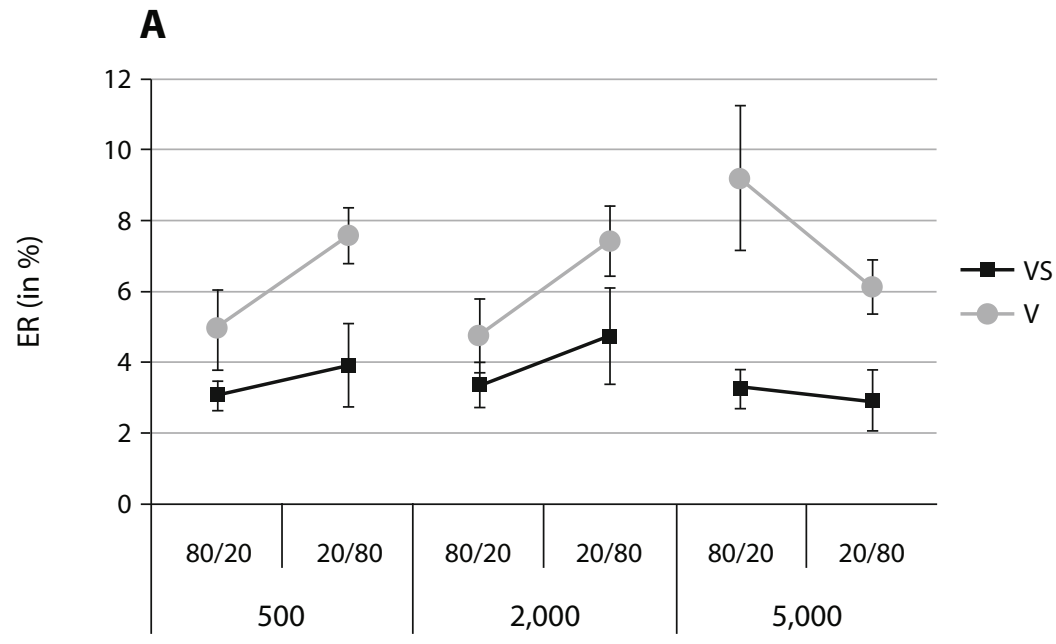

B

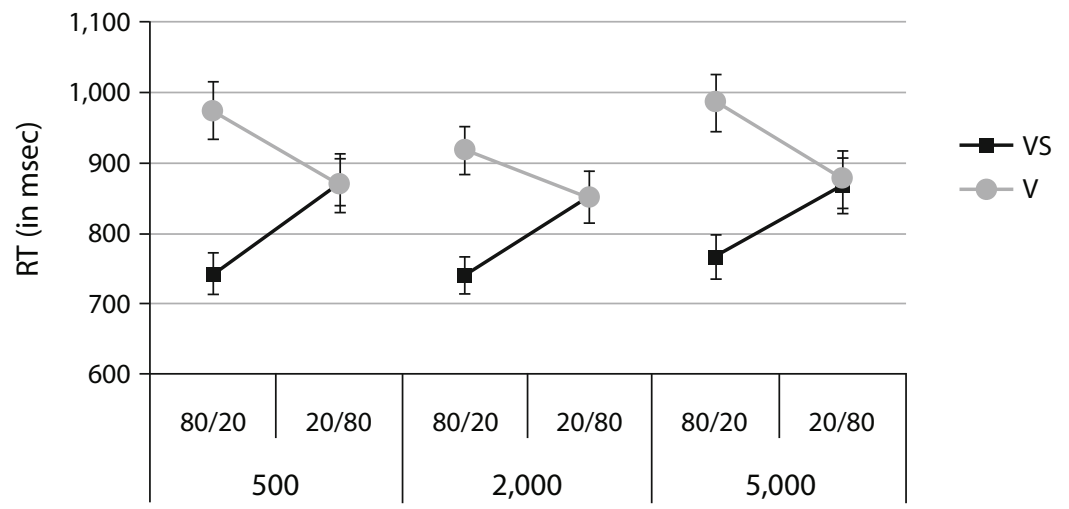

Figure 3. Experiment 2: (A) Mean error rate (ER) for the strategy analysis for each retention interval. (B) Mean response time (RT) for the strategy analysis for each retention interval. VS, visuospatial format; $V$, verbal format; $80 / 20,80 \%$ visuospatial expectancy and $20 \%$ verbal expectancy; $20 / 80,20 \%$ visuospatial expectancy and $80 \%$ verbal expectancy. Error bars represent standard errors of the means. 
Table 2

Mean Error Rates (\%) and Response Times (in Milliseconds) for the 500-msec, 2,000-msec, and 5,000-msec Retention Intervals in Experiment 2

\begin{tabular}{|c|c|c|c|c|c|c|c|c|c|c|c|c|c|}
\hline \multirow[b]{3}{*}{ Format } & \multirow{3}{*}{$\begin{array}{l}\text { Visual } \\
\text { Field }\end{array}$} & \multicolumn{4}{|c|}{$500 \mathrm{msec}$} & \multicolumn{4}{|c|}{$2,000 \mathrm{msec}$} & \multicolumn{4}{|c|}{$5,000 \mathrm{msec}$} \\
\hline & & \multicolumn{2}{|c|}{ Error Rate } & \multicolumn{2}{|c|}{ Response Time } & \multicolumn{2}{|c|}{ Error Rate } & \multicolumn{2}{|c|}{ Response Time } & \multicolumn{2}{|c|}{ Error Rate } & \multicolumn{2}{|c|}{ Response Time } \\
\hline & & $M$ & SEM & $M$ & $S E M$ & $M$ & SEM & $M$ & SEM & $M$ & SEM & $M$ & SEM \\
\hline \multicolumn{14}{|c|}{$80 \%$ Visuospatial Expectancy $/ 20 \%$ Verbal Expectancy } \\
\hline \multirow[t]{2}{*}{ Visuospatial } & Left & 3.1 & 0.52 & 731.1 & 27.6 & 3.0 & 0.64 & 740.8 & 28.2 & 3.8 & 0.71 & 773.9 & 31.5 \\
\hline & Right & 3.0 & 0.62 & 751.0 & 29.0 & 3.7 & 0.72 & 737.2 & 27.3 & 2.8 & 0.74 & 758.1 & 30.5 \\
\hline \multirow[t]{2}{*}{ Verbal } & Left & 6.6 & 1.78 & $1,005.7$ & 42.7 & 7.0 & 1.71 & 947.6 & 34.7 & 11.2 & 3.28 & 996.6 & 41.1 \\
\hline & Right & 3.3 & 1.01 & 945.3 & 42.5 & 2.5 & 1.10 & 900.7 & 39.5 & 7.3 & 1.83 & 974.5 & 42.1 \\
\hline \multicolumn{14}{|c|}{ 20\% Visuospatial Expectancy/80\% Verbal Expectancy } \\
\hline \multirow[t]{2}{*}{ Visuospatial } & Left & 3.0 & 1.55 & 875.2 & 37.6 & 5.8 & 1.83 & 843.4 & 41.4 & 2.6 & 1.11 & 859.4 & 39.1 \\
\hline & Right & 4.9 & 1.29 & 873.2 & 37.6 & 3.7 & 1.25 & 859.1 & 35.8 & 3.3 & 1.15 & 875.5 & 42.9 \\
\hline \multirow[t]{2}{*}{ Verbal } & Left & 9.0 & 1.24 & 870.0 & 38.6 & 8.7 & 1.36 & 869.3 & 39.1 & 6.6 & 1.14 & 899.1 & 42.5 \\
\hline & Right & 6.2 & 0.85 & 868.8 & 38.5 & 6.2 & 0.92 & 835.9 & 37.2 & 5.6 & 0.96 & 856.6 & 42.0 \\
\hline
\end{tabular}

interval $\times$ format $\times$ visual field $[F(2,21)=8.18, p<$ $.01]$. Follow-up tests for each separate retention interval showed significant effects of format and of expectancy $X$ format for all three intervals ( $p<.001$ for all effects). A significant interaction was found for format $\times$ visual field in both the 500-msec $(p<.05)$ and 2,000-msec $(p<$ $.05)$ intervals, whereas a significant interaction of expectancy $\times$ format $\times$ visual field was found for the 500 -msec $(p=.01)$ and the 5,000-msec $(p=.05)$ intervals. Followup paired-sample $t$ tests showed an LVF/RH advantage for the 500-msec interval with visuospatial expectancy and visuospatial format condition; and an RVF/LH advantage was found for the 500-msec interval with visuospatial expectancy and verbal format condition, as well as for the verbal expectancy and verbal format condition for both the 2,000-msec and 5,000-msec intervals.

\section{Discussion}

To further investigate differences in lateralization between verbally and visuospatially represented categories, the expectancy of the format of the second stimulus was manipulated in this experiment. The analysis of expectancy and format in RTs supports the use of a full strategic approach in the cross-dot task; for both formats, RTs were significantly lower when the expected format was actually presented. This means that subjects used different approaches when they expected verbal or visuospatial stimuli, and they adopted a more suitable strategy according to their expectation. Whereas self-reports on the original version of the task (van der Ham et al., 2007) indicated a general shift in the type of strategy used as the retention interval increased, the pattern of responses in the present version was comparable for all three retention intervals. A closer look at the interaction of expectancy, format, and interval indicated that only visuospatial expectancy with a verbal format was affected by interval. In this case, there was an increase in RTs for the longest interval. One possible explanation for this effect is that the visuospatial strategy is slightly more rigid for longer intervals and therefore leads to somewhat longer RTs when an unexpected verbal stimulus is shown.

The finding of a full strategic approach does not concur with the dual representational model suggested by
Noordzij et al. (2005). An important difference between the two experimental designs that might explain the different response pattern is the format of the first stimulus. In the Noordzij et al. (2005) study, the first stimulus was always verbal, whereas in the present design, it was always visuospatial. It therefore seems likely that the propositional representation is present, regardless of expectancy, because of the verbal format of the first stimulus. In other words, reading a spatial verbal label might force propositional coding. A visuospatial first stimulus does not influence type of coding in this way, however, because it does not lead to an overall presence of a pictorial representation.

As in Experiment 1, we examined the lateralization patterns by means of a general linear model. When the interval was brief $(500 \mathrm{msec})$ and a visuospatial stimulus was expected and presented, there was a significant right-hemisphere advantage. Three other effects showed a significant left-hemisphere advantage: when a verbal stimulus was expected and presented with a 2,000-msec interval, when it was expected and presented with a 5,000-msec interval, and when a visuospatial stimulus was expected and a verbal stimulus was shown for the 500 -msec interval. This illustrates a dissociation of verbal and visuospatial formats with regard to hemispheric advantage, which is partially in line with the Kemmerer and Tranel (2000) proposal; however, this effect is limited to visuospatial expectancy and the brief interval. It shows that task-driven strategy selection does not determine direction of lateralization - since the expectancy is the same in both cases - but lateralization is determined by the format of the second stimulus. A visuospatial strategy leads to a left-hemisphere advantage when a verbal stimulus is expected, with a brief interval. The other lateralization effect indicates that with the long interval, there is a lefthemisphere advantage related to verbal strategy, which is caused by verbal expectancy, and verbal stimulus format. Taken together, this means that regardless of task-driven strategy, a left-hemisphere advantage emerged only when the format was verbal. A critical contribution of merely reading the stimulus to the left-hemisphere advantage seems unlikely, because we did not find such an advantage in all verbal conditions. 


\section{GENERAL DISCUSSION}

Hemispheric lateralization of coordinate and categorical spatial relation processing has been addressed in a vast number of studies. The coordinate right-hemisphere advantage has been substantiated convincingly, whereas on some occasions the left-hemisphere advantage for categorical relations has been limited or absent. In two experiments, we further examined the lateralization of categorical processing when two different stimulus formats are involved: verbal and visuospatial. Although the traditional view of spatial relation processing does not provide a clear answer to the inconsistent findings for spatial category processing, Kemmerer and Tranel (2000) tried to do so with a case study report. They suggested that only clearly verbal categories show a left-hemisphere advantage, whereas visuospatial categories are related to a right-hemisphere advantage. The experiments discussed here were designed to contribute to this debate.

First, the variability of lateralization in the outcomes of the two present experiments is in line with previous observations of inconsistencies in lateralization patterns of categorical relation processing. In Experiments 1 and 2, a left-hemisphere advantage was found clearly only for verbal stimuli. A right-hemisphere advantage was found in one particular condition in the Experiment 2, in which subjects expected and were presented a stimulus in the visuospatial format with a brief retention interval. Similarly, the data pattern of Experiment 1 pointed toward a small right-hemisphere advantage for the visuospatial stimuli with the brief interval. One feasible explanation for the right-hemisphere advantage with the brief interval is the influence of coordinate representation. Every visuospatial stimulus contains categorical as well as coordinate information. Even though subjects were instructed to focus on only the categorical characteristics, coordinate information can also be used to memorize dot position and determine the category. That this coordinate influence was found only for the brief interval can be explained by the relatively fast decay of coordinate information; they are present less or are absent in longer intervals. Possibly, subjects rely on categorical as well as coordinate representations to some extent when they expect and are presented a visuospatial stimulus with a brief retention interval. One reason a right-hemisphere advantage was not found for categorical tasks in our previous studies could be that the present task design explicitly emphasized two different approaches to solving the task. The task entailed a clear separation of verbal and visuospatial characteristics. Since subjects were aware of these two different stimulus types and different levels of expectations, this may have led them to solve this condition completely pictorially. The pictorial approach could include some coordinate processing, which is strongly related to the right hemisphere. This is in line with the flexibility that subjects have shown in adapting to expectancy; they can clearly adjust their strategy in accordance with task demands.

An additional left-hemisphere advantage caused by reading could have been the main factor in the lefthemisphere advantages found in Experiment 1, but the design of Experiment 2 allowed us to examine this possibility. The data indicated that even when a visuospatial task-driven strategy was used, a left-hemisphere advantage was found for verbal stimuli. Importantly, only the format of the stimuli appears to have determined the lateralization effect. Reading could therefore still be a contributing factor with regard to left lateralization. It cannot explain the lateralization pattern completely by itself, however, since we did not find this pattern for all conditions with a verbal format. This finding has clear implications for the design of future studies. It indicates that the verbal nature of a task can influence the lateralization found; therefore, characteristics like the formulation of instructions, the showing of pictures versus explicitly naming the categories or the stimuli used, and the use of difficult- versus easy-to-verbalize stimuli can all be of critical influence. They can either eliminate lateralization or evoke a clear left-hemisphere advantage, respectively.

A variation in verbal characteristics may have been present in the large number of tasks that have tested categorical relation processing. On the basis of the present outcomes, differential verbal characteristics could well explain the variance in the hemispheric advantages reported. One note should be made here with regard to designing visuospatial categorical tasks: It appears nearly impossible to create a task without any verbal characteristics, because it is inherent in the definition of categorical spatial relations that they can be adequately described by prepositions, unlike in coordinate relations. One risk of attempting to make a fully nonverbal task could be that coordinate relation processing becomes vital to solving the task, which could explain any right-hemisphere advantage, such as that described by Kemmerer and Tranel (2000).

With regard to our examination of strategy in Experiment 2, we have found evidence for a full strategic approach, in contrast to the dual representational model-including propositional coding regardless of expectancy - suggested by Noordzij et al. (2005). In our study, subjects adapted fully to both visuospatial and verbal expectancy, without evidence of such a propositional coding effect. One possible explanation is that using a picture instead of a sentence as the first stimulus reduces the impact on which types of mental codes will be generated subsequently. Also, this strategic approach was similar in each retention interval. This last result does not completely concur with the selfreports for the original version of the task (van der Ham et al., 2007), in which we reported a change from a more visuospatial to a more verbal strategy when retention intervals increased in duration. An important distinction between that study and the present one should be mentioned here: In the present study, the task design was the determining factor in what strategy subjects employed, and the data were pooled over subjects. In the original task, however, we reported the naturally occurring strategy preferences by subjects. A pivotal next step would be to compare groups with different strategy preferences and see how they differ in lateralization patterns.

In short, we found a right-hemisphere advantage and multiple left-hemisphere advantages in some of the 
conditions. The right-hemisphere advantage cannot be explained by the original Kosslyn (1987) view, because that view states an overall left-hemisphere advantage of categorical processing regardless of format. In contrast, it appears to fit with Kemmerer and Tranel's (2000) proposal, since the left-hemisphere advantages are limited to verbal categories and the right-hemisphere advantage is present in a clearly visuospatial condition. The lefthemisphere advantage for verbal stimulus formats when a visuospatial strategy is adopted cannot by explained by the Kemmerer and Tranel view, however. A compromise between the two views seems in order, therefore. In our study, the main determinant in significance and direction of lateralization was the format of the second stimulus. As stated before, this emphasizes the importance of task design when investigating such lateralization effects. Consequently, the lateralization differences in Kemmerer and Tranel's data most likely did not reflect two different types of processing, but instead reflected the effect of verbal processing versus adding coordinate processing to categorical tasks. We believe that explicitly stated verbal processes can influence the lateralization outcome, but for different reasons than those given by Kemmerer and Tranel. The influence of verbal processes appears to be caused primarily by additional verbal characteristics affecting categorical processing, instead of by verbal versus visuospatial categorical processing. Additionally, reducing the possibility of verbal processing during categorical tasks too much might unintentionally allow for a considerable contribution of coordinate processing. We therefore recommend reducing the level of verbal characteristics of a task design to decrease these interfering verbal left hemisphere effects, occluding the spatial lateralization effects aimed for in those measurements. Likewise, categorical tasks should be clearly categorical and not require or even allow for strong coordinate processing, in order to avoid right-hemisphere processing overshadowing the left-hemisphere categorical activity.

\section{AUTHOR NOTE}

This study was supported by Evolution and Behaviour Grant 051-14027 from the Netherlands Organization for Scientific Research (NWO). We thank Monique te Beek, Karin Gerrits, and Uti Vidya for their help in gathering data for Experiment 1. Correspondence concerning this article should be addressed to I. J. M. van der Ham, Heidelberglaan 2, 3584 CS Utrecht, The Netherlands (e-mail: c.j.m.vanderham@uu.nl).

\section{REFERENCES}

Bourne, V. J. (2006). The divided visual field paradigm: Methodological considerations. Laterality, 11, 373-393.

Carpenter, P. A., \& Just, M. A. (1975). Sentence comprehension: A psycholinguistic processing model of verification. Psychological Review, 82, 45-73.

Chase, W. G., \& Clark, H. H. (1972). Mental operations in the comparison of sentences and pictures. In L. W. Gregg (Ed.), Cognition in learning and memory (pp. 205-232). New York: Wiley.

Clark, H. H., \& Chase, W. G. (1972). On the process of comparing sentences against pictures. Cognitive Psychology, 3, 472-517.

Hellige, J. B., \& Michimata, C. (1989). Categorization versus dis- tance: Hemispheric differences for processing spatial information. Memory \& Cognition, 17, 770-776.

Huttenlocher, J., Hedges, L.V., \& Duncan, S. (1991). Categories and particulars: Prototype effects in estimating spatial location. Psychological Review, 98, 352-376.

JAGER, G., \& Postma, A. (2003). On the hemispheric specialization for categorical and coordinate spatial relations: A review of the current evidence. Neuropsychologia, 41, 504-515.

Kemmerer, D., \& Tranel, D. (2000). A double dissociation between linguistic and perceptual representations of spatial relationships. $\mathrm{Cog}$ nitive Neuropsychology, 17, 393-414.

KossLyn, S. M. (1987). Seeing and imagining in the cerebral hemispheres: A computational approach. Psychological Review, 94, 148175.

Kosslyn, S. M., Koenig, O., Barrett, A., Cave, C. B., Tang, J., \& Gabrieli, J. D. E. (1989). Evidence for two types of spatial representations: Hemispheric specialization for categorical and coordinate relations. Journal of Experimental Psychology: Human Perception \& Performance, 15, 723-735.

Laeng, B., Chabris, C. F., \& Kosslyn, S. M. (2003). Asymmetries in encoding spatial relations. In R. Hugdahl \& R. J. Davidson (Eds.), The asymmetrical brain (pp. 303-339). Cambridge, MA: MIT Press.

Laeng, B., \& Peters, M. (1995). Cerebral lateralization for the processing of spatial coordinates and categories in left- and right-handers. Neuropsychologia, 33, 421-439.

MacLeod, C. M., Hunt, E. B., \& Mathews, N. N. (1978). Individual differences in the verification of sentence-picture relationships. Journal of Verbal Learning \& Verbal Behavior, 17, 493-507.

Martin, R., Houssemand, C., Schiltz, C., Burnod, Y., \& AlexanDRE, F. (2008). Is there continuity between categorical and coordinate spatial relations coding? Evidence from a grid/no-grid working memory paradigm. Neuropsychologia, 46, 576-594.

NoordziJ, M. L., van der Lubbe, R. H. J., Neggers, S. F. W., \& Postma, A. (2004). Spatial tapping interferes with the processing of linguistic spatial relations. Canadian Journal of Experimental Psychology, 58, 259-271.

NoordziJ, M. L., van der Lubbe, R. H. J., \& Postma, A. (2005). Strategic and automatic components in the processing of linguistic spatial relations. Acta Psychologica, 119, 1-20.

OLDFIELD, R. C. (1971). The assessment and analysis of handedness: The Edinburgh inventory. Neuropsychologia, 9, 97-113.

Postma, A., Huntjens, R. J. C., Meuwissen, M., \& Laeng, B. (2006). The time course of spatial memory processing in the two hemispheres. Neuropsychologia, 44, 1914-1918.

Reichle, E. D., Carpenter, P. A., \& Just, M. A. (2000). The neural bases of strategy and skill in sentence-picture verification. Cognitive Psychology, 40, 261-295.

Roberts, M. J., Wood, D. J., \& Gilmore, D. J. (1994). The sentencepicture verification task: Methodological and theoretical difficulties. British Journal of Psychology, 85, 413-432.

Rybash, J. M., \& Hoyer, W. J. (1992). Hemispheric specialization for categorical and coordinate spatial representations: A reappraisal. Memory \& Cognition, 20, 271-276.

Tversky, B. (1969). Pictorial and verbal encoding in a short-term memory task. Perception \& Psychophysics, 6, 225-233.

TVERSKY, B. (1974). Retrieval of pictorial and verbal stimulus codes. Bulletin of the Psychonomic Society, 4, 580-582.

van der Ham, I. J. M., Raemaekers, M., van Wezel, R. J. A., OlekSIAK, A., \& Postma, A. (2009). Categorical and coordinate spatial relations in working memory: An fMRI study. Brain Research, 1297, 70-79.

van der Ham, I. J. M., van Wezel, R. J. A., Oleksiak, A., \& Postma, A. (2007). The time course of hemispheric differences in categorical and coordinate spatial processing. Neuropsychologia, 45, 2492-2498.

WERNER, S., \& DiEDRICHSEN, J. (2002). The time course of spatial memory distortions. Memory \& Cognition, 30, 718-730.

(Manuscript received May 31, 2009; revision accepted for publication January 6,2010 .) 